\title{
Low Frequency Scaling of the Mixed MFIE for Scatterers with a Non-Simply Connected Surface
}

\author{
I. Bogaert* \\ K. Cools* \\ F. P. Andriulli ${ }^{\dagger}$ \\ D. De Zutter ${ }^{*}$
}

\begin{abstract}
The Magnetic Field Integral Equation (MFIE) is a widely used integral equation for the solution of electromagnetic scattering problems involving perfectly conducting objects. It is usually discretized by means of RWG functions as both basis and test functions. This discretization of the MFIE is well-known for its good condition number. However, it is equally well-known for the inferior accuracy of its solution when compared to the Electric Field Integral Equation (EFIE). What is less-known is that this accuracy problem becomes even more serious when the frequency is lowered. Recently it has been proved that the so-called mixed discretization of the MFIE, also called 'mixed MFIE', eliminates this low-frequency accuracy problem on simply connected scatterers. The mixed MFIE utilizes the so-called Buffa-Christiansen or Chen-Wilton functions for testing. In this contribution, the low frequency behavior of the mixed MFIE is investigated for scatterers with a non-simply connected surface. An analysis shows the presence of an approximate nullspace in the mixed MFIE at low frequencies. This nullspace becomes exact when the frequency is zero. This behavior matches known results for the continuous MFIE. Numerical results are presented that confirm this analysis. Despite the approximate nullspace at low frequencies, numerical results indicate that the mixed MFIE still delivers accurate results for toroidal scatterers.
\end{abstract}

\section{INTRODUCTION}

When electromagnetic scattering problems involving perfectly conducting objects are considered, the goal is to find a suitable approximation $\boldsymbol{j}(\boldsymbol{r})$ of the electrical current that runs over the surface of the scatterer. The MFIE and EFIE are the predominant integral equations for doing this. However, if the obtained solution current is to be considered a physical solution to the problem, it must be subject to the following scaling law in the low-frequency limit

$$
\nabla \cdot \boldsymbol{j}(\boldsymbol{r}) \approx \mathcal{O}(\omega)
$$

This is true because the charge distribution $\rho(\boldsymbol{r})$ associated with the solution current is given by $\rho(\boldsymbol{r})=\frac{j}{\omega} \nabla \cdot \boldsymbol{j}(\boldsymbol{r})$. We know that in reality, the charge distribution should converge to the static value when the low-frequency limit is taken. There-

*Department of Information Technology (INTEC), Ghent University, Belgium, e-mail: Ignace.Bogaert@intec.UGent.be

†École nationale suprieure des télécommunications de Bretagne, Microwave Department, France fore the divergence of the solution current must become proportional to the frequency, again in the low-frequency limit. Currents that satisfy (1) will be called low-frequency stable. When an electromagnetic scattering problem is discretized, however, it is not trivial to automatically get solution currents that are low-frequency stable. Indeed, ordinarily one would expect that when a continuous problem such as an integral equation is discretized, the discretization error would contaminate any small $\mathcal{O}(\omega)$ contributions to the solution current. Yet these small contaminations have a large effect on the fields. As it turns out, the lowfrequency stability of the solution current, i.e. the absence of contamination, depends critically upon the discretization strategy [1].

For both the MFIE and EFIE, the solution current is usually expanded in a basis of RWG functions [2]. The testing of the EFIE is usually done by means of rotated RWGs, where 'rotated' means that the cross product with the surface normal was taken. This discretization strategy has proven very successful regarding the accuracy of the solution current. In addition, the solution current is provably low-frequency stable. For the MFIE, the testing is usually done with the RWGs (not rotated), leading to the standard MFIE. Although this leads to a very well-conditioned system, the accuracy of the solution current is generally worse than that of the EFIE. Also, the current solution of the standard MFIE is not low-frequency stable. Therefore the charge associated with this solution current diverges when the frequency goes to zero. This behavior is neither physical nor consistent with the EFIE solution. It also has deleterious repercussions on the normal component of the electric field, which diverges along with the charge.

Recently, however, a new testing scheme for the MFIE was presented [3]. Instead of using the familiar RWG functions for the testing, this scheme uses the rotated Buffa-Christiansen or Chen-Wilton functions $[4,5]$. In [1], it was shown that the solution current of this so-called mixed MFIE is lowfrequency stable, which is in stark contrast with the standard MFIE. However, the analysis in [1] is limited to scatterers with simply connected surfaces, since global loops are not taken into account.

In this contribution we will show that the current 
solution of the mixed MFIE is low-frequency stable also in the case of a non-simply connected scatterer. In addition, it will be shown that the mixed MFIE has an exact kernel at zero frequency. This deep property is shared with the continuous MFIE, as shown in [6]. Numerical results will be provided to corroborate these claims.

\section{INTEGRAL EQUATION AND SCAT- TERER}

Consider a closed PEC scatterer with surface $\Gamma$ and exterior normal $\hat{\boldsymbol{n}}$, embedded in a background medium with permittivity $\epsilon$ and permeability $\mu$. The scatterer is illuminated by an incident electromagnetic field $\left(\boldsymbol{e}^{i}, \boldsymbol{h}^{i}\right)$. Enforcing the boundary condition for the tangential trace of the magnetic field on $\Gamma$ leads to the (outer) MFIE for the induced current density $j$ :

$$
\begin{gathered}
\hat{\boldsymbol{n}} \times \boldsymbol{h}^{i}(\boldsymbol{r})=M[\boldsymbol{j}](\boldsymbol{r})=\left\{\frac{1}{2}-K\right\}[\boldsymbol{j}](\boldsymbol{r}) \\
:=\frac{1}{2} \boldsymbol{j}(\boldsymbol{r})-\hat{\boldsymbol{n}} \times \frac{1}{4 \pi} p \cdot v \cdot \int_{\Gamma} \nabla \frac{e^{-j k R}}{R} \times \boldsymbol{j}\left(\boldsymbol{r}^{\prime}\right) d S^{\prime} .
\end{gathered}
$$

This equation can also be interpreted as: the magnetic field generated by the surface currents and the excitation must be zero just inside the boundary of the scatterer.

The first step in solving this equation is usually to approximate the surface $\Gamma$ by means of a mesh consisting of $V$ vertices, $E$ edges, and $F$ faces. In the case of a closed scatterer with genus $g$ (intuitively the number of holes in the scatterer), $V, E$ and $F$ are related by Euler's formula

$$
V+F-E=2-2 g .
$$

In the rest of this contribution, a torus-shaped scatterer will be used, hence $g=1$.

The second step in solving equation (2) is the discretization. Two sets of functions will be used in the rest of this paper: the familiar RWG functions [2] and the Buffa-Christiansen (BC) or ChenWilton functions $[4,5]$. Both these sets of functions have one function per edge of the mesh. When the MFIE (2) is discretized in the standard way, the RWG functions are used as both basis and test functions. In the mixed discretization of the MFIE, the testing functions are the rotated $\mathrm{BC}$ functions.

\section{SUBSPACES}

To demonstrate the low-frequency stability of the solution current in the mixed MFIE, the RWG and $\mathrm{BC}$ finite element spaces must each be split into two subspaces. The first subspace is the space of divergence-free functions, i.e. the so-called loops. The second subspace is simply defined as the complement of the loop space, and can for example be defined as the space of the so-called stars (see for example [7]). Throughout this contribution, the complementary space will be called the star space.

It is an easy matter to determine the sizes of the loop and star space. Indeed, because the loops do not generate any charges, the star space is responsible for generating the charge. In the RWG finite element space, there are $F-1$ independent charge degrees of freedom. Hence there are $F-1$ stars. The number of loops is the total number of edges $E$ minus the number of stars, i.e. $V+2 g-1$. For the $\mathrm{BC}$ finite element space, a similar reasoning can be made, with the difference that the $\mathrm{BC}$ charges are associated with the vertices of the mesh instead of the faces. Hence, there are $V-1 \mathrm{BC}$ stars and $F+2 g-1$ BC loops. The loop subspaces are, for the most part, composed of local loops. Local loops are loops that can be contracted to a point, i.e. they are not wrapped around one of the holes or handles of the scatterer. There are $V-1$ local RWG loops and $F-1$ local BC loops. Therefore, if the scatterer is simply connected, there are only local loops. However, if the scatterer is a torus (such that $g=1$ ), two additional loops appear in both the RWG and BC finite element spaces. In general, these two loops will both carry current around the hole as well as around the handle. By taking suitable linear combinations it is possible to construct a purely toroidal and a purely poloidal loop. The toroidal loop carries no net current in the poloidal direction and vice versa. These two special loops are well-studied in the literature and are visualized for example in Figure 4 in [6], with Fig 4(a) showing the toroidal loop and 4(b) showing the poloidal loop. In the following, the toroidal and poloidal loop will be separated from the loop space and be taken as separate subspaces. In this manner, four subspaces are present in both the RWG and BC finite element spaces: the stars, the local loops, the toroidal loop and the poloidal loop.

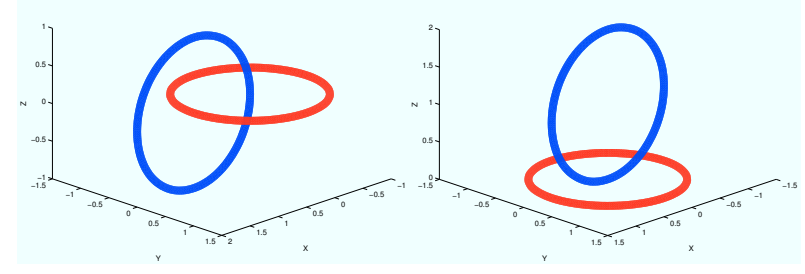

Figure 1: Loops that respectively pass and don't pass through each other. 


\section{LOW FREQUENCY STABILITY OF THE SOLUTION CURRENT}

Now consider the mixed discretization of the MFIE, after doing a suitable basis transformation to the subspaces described above:

$$
\underbrace{\left[\begin{array}{cccc}
\mathrm{M}^{s s} & \mathrm{M}^{s l} & \mathrm{M}^{s L_{t}} & \mathrm{M}^{s L_{p}} \\
\mathrm{M}^{l s} & \mathrm{M}^{l l} & \mathrm{M}^{L_{t}} & \mathrm{M}^{l L_{p}} \\
\mathrm{M}^{L_{t} s} & \mathrm{M}^{L_{t} l} & \mathrm{M}^{L_{t} L_{t}} & \mathrm{M}^{L_{t} L_{p}} \\
\mathrm{M}^{L_{p} s} & \mathrm{M}^{L_{p} l} & \mathrm{M}^{L_{p} L_{t}} & \mathrm{M}^{L_{p} L_{p}}
\end{array}\right]}_{\mathrm{M}} \cdot\left[\begin{array}{c}
\boldsymbol{I}^{s} \\
\boldsymbol{I}^{l} \\
\boldsymbol{I}^{L_{t}} \\
\boldsymbol{I}^{L_{p}}
\end{array}\right]=\left[\begin{array}{c}
\boldsymbol{G}^{s} \\
\boldsymbol{G}^{l} \\
\boldsymbol{G}^{L_{t}} \\
\boldsymbol{G}^{L_{p}}
\end{array}\right]
$$

where

$$
\begin{aligned}
\mathrm{M}^{\mathcal{V W}} & =\left\langle\hat{\boldsymbol{n}} \times \mathrm{BC} \mathcal{V} \text { space }\left|\frac{1}{2}-K\right| \operatorname{RWG} \mathcal{W} \text { space }\right\rangle, \\
\boldsymbol{G}^{\mathcal{V}} & =\left\langle\hat{\boldsymbol{n}} \times \mathrm{BC} \mathcal{V} \text { space } \mid \hat{\boldsymbol{n}} \times \boldsymbol{h}^{i}(\boldsymbol{r})\right\rangle .
\end{aligned}
$$

The symbols $\mathcal{V}$ and $\mathcal{W}$ can be either 'star', 'local loop', 'toroidal loop' or 'poloidal loop', and when they occur as superscript (as in (4)) they are abbreviated to ' $s$ ', 'l', ' $L_{t}$ ' and ' $L_{p}$ ' respectively.

Now the low-frequency behavior of the various blocks in (4) will be investigated. As was mentioned in [1], the block $\mathrm{M}^{l l}$ scales as $\mathcal{O}\left(\omega^{2}\right)$ at low frequencies. For a proof we refer to [8]. For global loops, however, the proof given in [8] is not generally valid. However, it can be proven that the following rule applies to both local and global loops: when the basis loop does not pass through the test loop (as in the rightmost graph in Figure 1), the matrix element scales as $\mathcal{O}\left(\omega^{2}\right)$. Otherwise (as in the leftmost graph in Figure 1) the matrix element scales as $\mathcal{O}(1)$. Note that when the basis loop does not pass through the test loop, the test loop also does not pass through the basis loop, so the rule is symmetric with respect to basis and test loop.

For the investigation of the behavior of the blocks by means of this rule, it is very useful to use the interpretation of the MFIE as given in Section 2: the magnetic field generated by the surface currents and the excitation must be zero just inside the boundary of the scatterer. By this interpretation, the testing functions can be thought of as being slightly inside the boundary of the scatterer, while the basis functions are on the boundary. This interpretation, along with the rule introduced in the above, will allow an intuitive assessment of the scaling of the matrix elements.

First, the $\mathcal{O}\left(\omega^{2}\right)$ scaling of the $\mathrm{M}^{l l}$ block is easily reproduced. Indeed, the local basis loops reside on the surface and do not pass through any local test loops, since those are located slightly inside of the torus. The same reasoning holds for the blocks $\mathrm{M}^{L_{t} l}$ and $\mathrm{M}^{L_{p} l}$, since the toroidal and poloidal test loops never cross these local basis loops. Analogously, the blocks $\mathrm{M}^{l L_{t}}$ and $\mathrm{M}^{l L_{p}}$ scale as $\mathcal{O}\left(\omega^{2}\right)$ because the toroidal and poloidal basis loops never cross these local test loops. The $\mathrm{M}^{L_{p} L_{p}} \mathrm{M}^{L_{p} L_{t}}$ blocks also scale as $\mathcal{O}\left(\omega^{2}\right)$ because the poloidal test loop can never get passed through by a basis loop (since the test loop is slightly inside of the scatterer). The $\mathrm{M}^{L_{t} L_{t}}$ block also scales as $\mathcal{O}\left(\omega^{2}\right)$ because the basis loop does not carry a net current in the poloidal direction. Finally, the $\mathbf{M}^{L_{t} L_{p}}$ block does not scale as $\mathcal{O}\left(\omega^{2}\right)$ because the poloidal basis loop carries current trough the toriodal test loop. Therefore, this block scales as $\mathcal{O}(1)$, just like all the blocks involving stars. The excitation, when tested with a loop, yields a result of $\mathcal{O}(\omega)$ [8]. Summarizing these results, the following scaling is obtained for the matrix equation (4)

$\left[\begin{array}{cccc}\mathcal{O}(1) & \mathcal{O}(1) & \mathcal{O}(1) & \mathcal{O}(1) \\ \mathcal{O}(1) & \mathcal{O}\left(\omega^{2}\right) & \mathcal{O}\left(\omega^{2}\right) & \mathcal{O}\left(\omega^{2}\right) \\ \mathcal{O}(1) & \mathcal{O}\left(\omega^{2}\right) & \mathcal{O}\left(\omega^{2}\right) & \mathcal{O}(1) \\ \mathcal{O}(1) & \mathcal{O}\left(\omega^{2}\right) & \mathcal{O}\left(\omega^{2}\right) & \mathcal{O}\left(\omega^{2}\right)\end{array}\right] \cdot\left[\begin{array}{c}\boldsymbol{I}^{s} \\ \boldsymbol{I}^{l} \\ \boldsymbol{I}^{L_{t}} \\ \boldsymbol{I}^{L_{p}}\end{array}\right]=\left[\begin{array}{l}\mathcal{O}(1) \\ \mathcal{O}(\omega) \\ \mathcal{O}(\omega) \\ \mathcal{O}(\omega)\end{array}\right]$

From this result, the existence of a nullspace of the mixed MFIE on a torus can be immediately deduced. Indeed, consider the $V$-dimensional space $\mathcal{Q}$ of currents composed of local RWG loops and the toroidal global loop. The Mixed MFIE matrix applied to such a current yields

$$
\mathrm{M} \cdot\left[\begin{array}{c}
0 \\
\boldsymbol{I}^{l} \\
\boldsymbol{I}^{L_{t}} \\
0
\end{array}\right]=\left[\begin{array}{c}
\boldsymbol{v} \\
\mathcal{O}\left(\omega^{2}\right) \\
\mathcal{O}\left(\omega^{2}\right) \\
\mathcal{O}\left(\omega^{2}\right)
\end{array}\right]
$$

with

$$
\underbrace{\boldsymbol{v}}_{V-1 \text { by } 1}=\underbrace{\left[\begin{array}{ll}
\mathrm{M}^{s l} & \mathrm{M}^{s L_{t}}
\end{array}\right]}_{V-1 \text { by } V} \cdot \underbrace{\left[\begin{array}{c}
\boldsymbol{I}^{l} \\
\boldsymbol{I}^{L_{t}}
\end{array}\right]}_{V \text { by } 1}
$$

Because the matrix in the right hand side has one more column than it has rows, there exists (at least) one element of $\mathcal{Q}$ such that

$$
\left[\begin{array}{ll}
\mathrm{M}^{s l} & \mathrm{M}^{s L_{t}}
\end{array}\right] \cdot\left[\begin{array}{c}
\boldsymbol{I}_{0}^{l} \\
\boldsymbol{I}_{0}^{L_{t}}
\end{array}\right]=0,
$$

where the subscript 0 was given to this specific element of $\mathcal{Q}$. Now the following holds:

$$
\mathrm{M} \cdot\left[\begin{array}{c}
0 \\
\boldsymbol{I}_{0}^{l} \\
\boldsymbol{I}_{0}^{L_{t}} \\
0
\end{array}\right]=\left[\begin{array}{c}
0 \\
\mathcal{O}\left(\omega^{2}\right) \\
\mathcal{O}\left(\omega^{2}\right) \\
\mathcal{O}\left(\omega^{2}\right)
\end{array}\right]=\mathcal{O}\left(\omega^{2}\right)
$$


Obviously, this element becomes a null space when the frequency is dropped to zero. Figure 2 shows the numerically computed smallest singular value of the mixed MFIE matrix for a PEC torus (large radius $2 \mathrm{~m}$, small radius $1 \mathrm{~m}$, discretized with 676 faces) at various frequencies. The proportionality with $\omega^{2}$ is clearly visible.

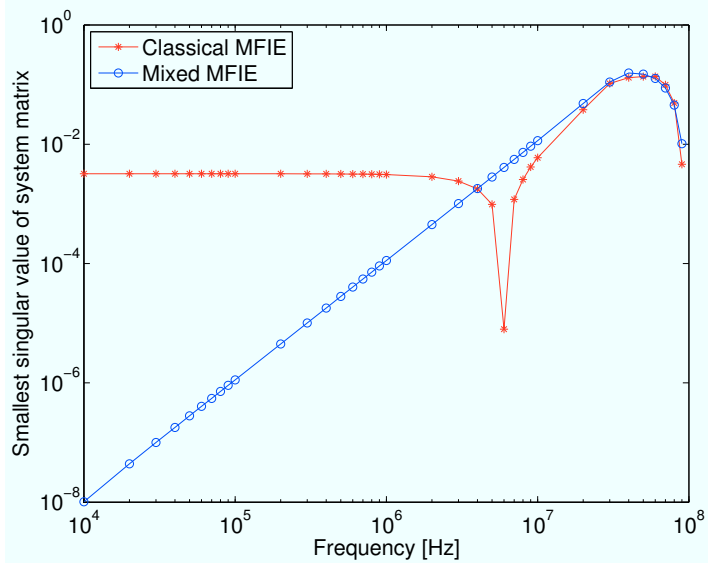

Figure 2: The smallest singular value of the system matrix as a function of the frequency for the standard and mixed MFIE.

At first sight, the presence of this nullspace would seem to jeopardize the low-frequency behavior of the solution current. However, this is not necessarily the case. Indeed, if the current in the nullspace is not excited by the source, then a well-behaved solution current may still be possible. In Figure 3, the norm of the electric field was computed in a ring-shaped set of points outside of the torus (the radius of the ring is $4 \mathrm{~m}$ ). These norms were all summed up to provide a crude measure for the magnitude of the electric field in the neighborhood of the torus. Apparently, the standard MFIE solution yields an electric field that diverges, while the EFIE and mixed MFIE both yield well-behaved fields.

\section{Acknowledgments}

The work of I. Bogaert was supported by a postdoctoral grant from the Fund for Scientific Research (Fonds Wetenschappelijk Onderzoek).

\section{References}

[1] I. Bogaert, K. Cools, F. P. Andriulli, J. Peeters, and D. De Zutter, "Low Frequency Stability of the Mixed Discretization of the MFIE," in Submitted to the 5th European Conference on Antennas and Propagation, Rome, Italy, 11-15 April 2011.

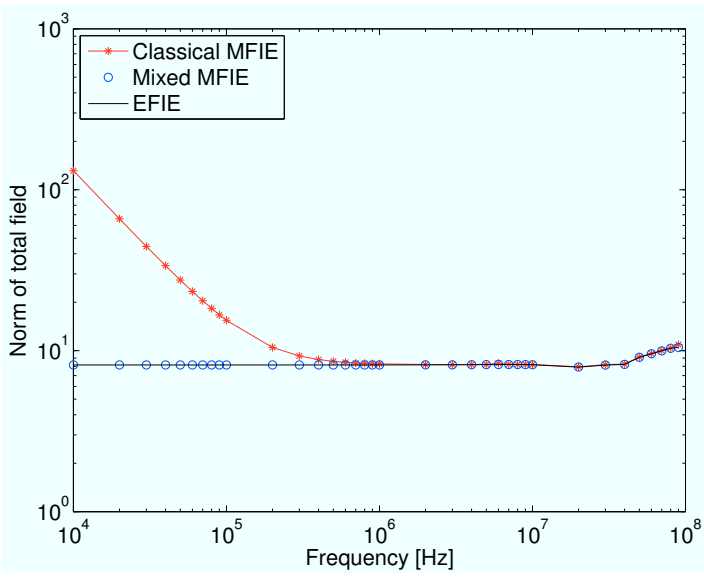

Figure 3: The norm of the electric field just outside the torus.

[2] S. D. Rao, D. R. Wilton, and A. W. Glisson, "Electromagnetic scattering by surfaces of arbitrary shape," IEEE Trans. Antennas Propag., vol. AP-30, no. 3, May 1982.

[3] K. Cools, F. Andriulli, F. Olyslager, and E. Michielssen, "Improving the MFIE's accuracy by using a mixed discretization," in Antennas and Propagation Society International Symposium, June 2009, pp. 1-4.

[4] A. Buffa and S. H. Christiansen, "A dual finite element complex on the barycentric refinement," Math. of Comp., vol. 260, pp. 17431769, 2007.

[5] Q. Chen and D. Wilton, "Electromagnetic scattering by threedimensional arbitrary complex material/conducting bodies," Proceedings of the IEEE Symposium on Antennas and Propagation, vol. 2, pp. 590-593, 1990.

[6] K. Cools and F. P. Andriulli and F. Olyslager and E. Michielssen, "Nullspaces of MFIE and Calderon Preconditioned EFIE Operators Applied to Toroidal Surfaces," IEEE Transactions on Antennas and Propagation, vol. 57, no. 10, pp. 3205-3215, Oct 2009.

[7] G. Vecchi, "Loop-star decomposition of basis functions in the discretization of the efie," IEEE Transactions on Antennas and Propagation, vol. 47, no. 2, pp. 339-346, Feb 1999.

[8] S. Chen, W. Chew, J. Song, and J.-S. Zhao, "Analysis of low frequency scattering from penetrable scatterers," IEEE Transactions on Geoscience and Remote Sensing, vol. 4, no. 39, p. 726, 7352001. 